
$\$$ Research Square
Preprints are preliminary reports that have not undergone peer review.
They should not be considered conclusive, used to inform clinical practice, or referenced by the media as validated information.

\title{
The effect of prenatal and postnatal treatment with intravenous immunoglobulin on severity of neonatal hemochromatosis: the tale of two brothers (case report)
}

Veronica Mugarab Samedi ( $\sim$ vmsamedi@gmail.com )

University of Saskatchewan https://orcid.org/0000-0002-7697-0893

Michelle D Ryan

University of Calgary Cumming School of Medicine

Essa Al Awad

University of Calgary Cumming School of Medicine

Adel Elsharkawy

University of Calgary Cumming School of Medicine

\section{Case report}

Keywords: Neonatal hemochromatosis, Intravenous immunoglobulin, prenatal treatment

Posted Date: January 17th, 2020

DOl: https://doi.org/10.21203/rs.2.21146/v1

License: (c) (1) This work is licensed under a Creative Commons Attribution 4.0 International License.

Read Full License 


\section{Abstract}

Background: Neonatal hemochromatosis $(\mathrm{NH})$ is a rare condition that was the main reason for liver transplantation in infants. With the realization that $\mathrm{NH}$ results from the fetal complement-mediated liver injury, intravenous immunoglobulins (IVIG) were successfully introduced for the treatment.

Case Presentation: We present two cases of $\mathrm{NH}$ from the same family to illustrate the role of antenatal treatment with IVIG in alleviation and possible prevention of this serious morbidity.

Conclusion: A prenatal treatment and early postnatal administration of IVIG are effective ways to manage $\mathrm{NH}$ that help to reduce the severity of the symptoms, prevent liver failure and avoid the need for liver transplantation Keywords: Neonatal hemochromatosis, Intravenous immunoglobulin, prenatal treatment

\section{Background:}

Neonatal hemochromatosis $(\mathrm{NH})$ is a rare condition that presents with early liver failure accompanied by hepatic and extrahepatic iron deposition (sideration) ${ }^{1}$. For many years $\mathrm{NH}$ was the main reason for liver transplantation in infants; supportive treatment with antioxidants and chelation was ineffective and associated with severe side effects ${ }^{1-3}$. With the realization that $\mathrm{NH}$ results from the fetal complementmediated liver injury, intravenous immunoglobulins (IVIG) were successfully introduced for the treatment that subsequently resulted in increased survival rate and decreased need for liver transplantation ${ }^{3.4}$.

We present two cases of $\mathrm{NH}$ from the same family to illustrate the role of antenatal treatment with IVIG in alleviation and possible prevention of this serious morbidity.

Case presentation:

Case 1

The mother of our patient was an asymptomatic gravida 4 para 2 woman with two previous term deliveries and one miscarriage at 12 weeks. She had regular prenatal follow up, her antenatal screening showed normal serology, and she denied any history of illnesses during pregnancy. Current pregnancy was uneventful until 28 weeks of gestational age (GA) when fetal ultrasound (US) showed severe oligohydramnios that rapidly progressed to the anhydramnios despite intact membranes. The infant was delivered by emergency caesarian section at 29 weeks due to BPP 0/8 and poor fetal tracing. Apgar scores at birth were 3,7 and 7 at 1 st, 5 th and 10th minutes, respectively. The infant weighed 840 grams (8th percentile) and his initial physical examination was appropriate for the gestational age. He was intubated in the case room and received a surfactant for respiratory distress. During the next 24 hours, he remained on ventilatory support, and gradually developed hypoglycemia that was managed with glucose infusion. On his second day of life, the infant developed severe hypotension, thrombocytopenia, and progressive hypoxemia. His hypoglycemia precipitously worsens in spite of the increased glucose infusion rate (GIR). During this prolonged hypoglycemic episode a critical blood testing (insulin, cortisol, growth hormone, thyroid function, and lactate), a coagulation panel, and tests for possible metabolic 
abnormalities were performed as well as a full sepsis workup (complete blood count, C-reactive protein level, blood culture, and cerebrospinal fluid analysis and culture). Treatment with antibiotics (ampicillin and gentamycin) was initiated. Initial blood tests showed leukopenia with normal platelets count, elevated liver enzymes, and evidence of coagulopathy. The next day, the patient's condition deteriorated. He developed progressive abdominal distension with clinical picture of ileus, his liver function precipitously worsened; coagulation tests showed disseminated intravascular coagulation (DIC) despite treatment with fresh frozen plasma, cryoprecipitate, platelets and vitamin K. His ferritin level was very high $(2750 \mathrm{ng} / \mathrm{mL})$, and he continues to have profound lactic acidosis (lowest lactate level was 7. $2 \mathrm{mmol} / \mathrm{L}$ and highest $15.1 \mathrm{mmol} / \mathrm{L}$ ). The metabolic workup was reported as normal. Exploration laparotomy was performed for suspected necrotic bowel and showed mild ascites with multiple hemorrhages within the bowel wall. At the age of 3 days, the patient developed generalized tonic-clonic seizures that were refractory to the Phenobarbital, Phenytoin, and Levetiracetam. The patient remained hemodynamically unstable despite aggressive resuscitation and died at the age of 4 days.

An autopsy was performed and revealed abnormal, cirrhotic liver with nodular appearance, cholestasis and iron deposition in hepatocytes and Kupffer cells. Iron staining was found in thyroid, pancreas, mucous glands of upper respiratory tract and thymus. Hemorrhage within the lungs, cerebellum, lateral ventricles, subarachnoid were reported as well. Autopsy findings were consistent with the diagnosis of neonatal hemochromatosis $(\mathrm{NH})$.

\section{Case 2}

After confirming the diagnosis of $\mathrm{NH}$, parents were counseled about the risks of morbidity in the next pregnancy and explained that the probability of the next infant to be lethally affected is greater than $90 \%$. However, they were informed that the severity of $\mathrm{NH}$ could be considerably alleviated by antenatal IVIG treatment.

The next spontaneous singleton pregnancy occurred 26 months later, and the mother was under close surveillance of high-risk maternity services from 10 weeks of gestation. Preventive treatment with IVIG was initiated at 14 weeks of pregnancy until the end of gestation. The pregnancy was uneventful, and multiple fetal ultrasounds were reassuring. At $39+5$ weeks of GA, a male infant was born by spontaneous vaginal delivery. Apgar scores at birth were 7 and 9 at 1 st and 5th minutes, respectively. The infant weighed 3275 grams, and his physical examination was unremarkable. He was admitted to NICU for the management of possible neonatal hemochromatosis. His course at NICU was notable for transient hypoglycemia that was treated with intravenous glucose and moderately deranged coagulation profile: low fibrinogen, slightly increased INR, and elevated ferritin $(950 \mathrm{ng} / \mathrm{mL})$. Although the patient remained asymptomatic, treatment with IVIG $1 \mathrm{~g} / \mathrm{kg}$ was started immediately. Vitamin E 40 units twice a day was added after consultation with a pediatric gastroenterologist. Following treatment, the patient showed rapid laboratory improvement: fibrinogen ( $90 \mathrm{mg} / \mathrm{dL}$ to $175 \mathrm{mg} / \mathrm{dL}$ ), INR (2.68 to 1.1), and ferritin $(950 \mathrm{ng} / \mathrm{mL}$ to $510 \mathrm{ng} / \mathrm{mL})$. The patient was discharged home on day 20 of life with planned follow-up with pediatrician and gastroenterologist.

\section{Discussion And Conclusion}


$\mathrm{NH}$ is a rare but often life-threatening clinical condition, and untreated it is uniformly lethal ${ }^{1-3}$. Although the inheritance of $\mathrm{NH}$ remains unclear, the recurrence of $\mathrm{NH}$ in families with one affected child is about $90 \% 3,5$. Current clinical evidence explains $\mathrm{NH}$ as a maternofetal alloimmune disorder, so this condition is congenital but not hereditary ${ }^{3,4}$. NH starts at 14 weeks of pregnancy when maternal IgG began to cross placenta and bind to fetal hepatocytes causing complement-mediated hepatocyte injury ${ }^{5-7}$. The discovery of the alloimmune etiology of $\mathrm{NH}$ advanced prevention, timely diagnosis, and treatment of this condition 5.6 .

It is well described in the literature that a woman could have multiple unaffected infants before having an infant with $\mathrm{NH}^{1-3,6,7}$. In our case, the mother had two healthy children before the miscarriage, and afterward, an infant with severe $\mathrm{NH}$ was born. This fact could be explained by the time-lapse between exposure, sensitization, and production of IgG antibodies against the fetal antigen ${ }^{7,8}$.

While the majority of $\mathrm{NH}$ affected infants present with acute liver failure within hours after delivery, some could have isolated symptoms of hypoglycemia, coagulopathy, or hyperbilirubinemia at birth ${ }^{7.9}$. Without early and aggressive medical treatment these infants usually deteriorate within the first week of life, $\mathrm{e}^{10,11}$. The intrauterine growth restriction, oligohydramnios, prematurity are common co-foundings in infants with $\mathrm{NH}^{11,12}$.

This diagnosis without family history could be a challenge. Laboratory evaluation is usually significant for hyperbilirubinemia, hypoglycemia, and deranged coagulation factors. Iron studies reveal high serum ferritin levels $\left(>800 \mathrm{ng} / \mathrm{mL}\right.$ ) which is a sensitive but not specific indicator for $\mathrm{NH}^{1,5,11}$. Placental pathology findings are none-specific for $\mathrm{NH}$, and consist of edematous placental villi ${ }^{2}$.

In the first presented case combination of being small-for-gestational-age, having highly elevated ferritin levels, and liver failure made the diagnosis for $\mathrm{NH}$ most likely, and buccal biopsy was considered as a confirmation test, however, rapid clinical deterioration makes it unfeasible. The autopsy confirmed the suspected diagnosis of $\mathrm{NH}$ and provided with the necessary information for further counseling.

Since the Introduction of IVIG preventive therapy, mortality and morbidity rate among neonates with $\mathrm{NH}$ decreased dramatically $7,8,9$. Feldman and Whitington (2013) developed an effective protocol of $\mathrm{NH}$ prevention, with the use of IVIG at 14 weeks, 16 weeks, and 18 weeks, and then weekly until the end of pregnancy in mothers with a history of fetal or neonatal hemochromatosis ${ }^{3}$. They reported good outcomes in $99 \%$ of cases $^{3}$. Several studies support this approach and report similar effectiveness ${ }^{6.8 .9}$. In situations when mothers were unable to receive preventive therapy with IVIG, exchange transfusion, IVIG, and chelation-antioxidant therapy remain the treatment choices for the neonates with $\mathrm{NH}^{5,9}$.

In our second case, the mother received preventive therapy with IVIG that helped to preserve pregnancy until term (previously, she had a miscarriage and preterm birth at 29 weeks). This infant was born appropriate for gestational age and was clinically asymptomatic. His laboratory findings (elevated ferritin, deranged coagulation profile) on the first day of life were suggestive of impaired liver function, so, 
IVIG was administrated aiming to reduce the ongoing hepatocyte injury. We believe that the early use of IVIG in this patient helped to preserve hepatocytes and normalize liver function. His hospital course was straightforward, he was observed for 3 weeks and discharged home with no active concerns.

A prenatal treatment and early postnatal administration of IVIG are effective ways to manage $\mathrm{NH}$ that help to reduce the severity of the symptoms, prevent liver failure and avoid the need for liver transplantation.

\section{Abbreviations}

Congenital hepatoblastoma (CH), Neonatal Intensive Care Unit (NICU), Magnetic Resonance Imaging (MRI)

\section{Declarations}

Declarations: Ethics approval and consent to participate: no ethics approval required, informed consent from the patient received

Consent for publication: Written informed consent was obtained from the patient's parents for publication of this case report and any accompanying images. A copy of the written consent is available for review by the Editor-in-Chief of this journal.

Availability of data and material: available for review

Competing interests: no competing interests

Funding: not funded

\section{Authors' contributions:}

VMS- informed consent, data collection, literature review, manuscript preparation, direct involvement in patient care

MR: initial draft of manuscript, literature review, direct involvement in patient care

EA: manuscript edition, direct involvement in patient care

AE: manuscript edition, direct involvement in patient care

All authors have read and approved the manuscript.

Acknowledgements: Neonatal Intensive Care medical team Foothills Medical Centre, Calgary, Canada

\section{References}


1. Murray KF, Kowdley KV . Neonatal hemochromatosis. Pediatrics 2001; 108 (4): 960-964.

2. Sigurdsson L, Reyes J, Kocoshis SA, Hansen TW, Rosh J, Knisely AS . Neonatal hemochromatosis: outcomes of pharmacologic and surgical therapies. J Pediatr Gastroenterol Nutr 1998; 26 (1): 8589.

3. Feldman A.G , Whitington P.F. Neonatal Hemochromatosis. J Clin Exp Hepatol. 2013 Dec; 3(4): 313320. doi: 10.1016/j.jceh.2013.10.004

4. Taylor SA, Kelly S, Alonso EM, Whitington PF. The Effects of Gestational Alloimmune Liver Disease on Fetal and Infant Morbidity and Mortality. J Pediatr. 2018 May;196:123-128.e1. doi: 10.1016/j.jpeds.2017.12.054

5. Heissat S, Collardeau-Frachon S, Baruteau J et al. Neonatal hemochromatosis: diagnostic work-up based on a series of 56 cases of fetal death and neonatal liver failure. J Pediatr. 2015 Jan;166(1):6673. doi: $10.1016 /$ j.jpeds.2014.09.030

6. Rand EB, Karpen SJ, Kelly S, Mack CL, Malatack JJ, Sokol RJ et al. Treatment of neonatal hemochromatosis with exchange transfusion and intravenous immunoglobulin. J Pediatr 2009; 155 (4): 566-571.

7. Whitington and J. U. Hibbard, "High-dose immunoglobulin during pregnancy for recurrent neonatal haemochromatosis," The Lancet, vol. 364, pp. 1690-1698, 2004.

8. Pan X., Kelly S., Melin-Aldana H., Malladi P., Whitington P.F. Novel mechanism of fetal hepatocyte injury in congenital alloimmune hepatitis involves the terminal complement cascade. Hepatology. Jun 2010;51(6):2061-2068

9. Whitington P.F., Kelly S. Outcome of pregnancies at risk for neonatal hemochromatosis is improved by treatment with high-dose intravenous immunoglobulin. Pediatrics. Jun 2008;121(6):e1615-1621.

10. Tanaka H, Haba R, Itoh S, Sakamoto H, Hata T. Prenatal high-dose immunoglobulin treatment for neonatal hemochromatosis: a case report and review of the literature. J Obstet Gynaecol Res. 2011 Dec;37(12):1891-4. doi: 10.1111/j.1447-0756.2011.01680.x

11. Jimenez-Rivera C, Gupta A, Feberova J, de Nanassy JA, Boland MP. Successful treatment of neonatal hemochromatosis as gestational alloimmune liver disease with intravenous immunoglobulin. $\mathrm{J}$ Neonatal Perinatal Med. 2014;7(4):301-4. doi: 10.3233/NPM-14814026.

12. Babor, B. Hadzik, H. Stannigel, E. Mayatepek, and T. Hoehn, "Successful management of neonatal hemochromatosis by exchange transfusion and immunoglobulin: a case report," Journal of Perinatology, vol. 33, no. 1, pp. 83-85, 2013. 